\title{
Manejo de pacientes com Transtorno Depressivo Maior no contexto da Atenção Primária à Saúde: uma revisão de literatura
}

\author{
Management of patients with Major Depressive Disorder in the context of Primary Health Care: a
} literature review

Manejo de pacientes com Transtorno Depressivo Mayor en el contexto de la Atención Primaria de

Salud: revisión de la literatura

Recebido: 09/01/2022 | Revisado: 14/01/2022 | Aceito: 16/01/2022 | Publicado: 18/01/2022

\author{
Rafael Vieira Sampaio \\ ORCID: https://orcid.org/0000-0002-5774-4663 \\ Universidade do Estado do Pará, Brasil \\ E-mail: rafaelsampaio.med@hotmail.com \\ Paulo Humberto Mendes de Figueiredo \\ ORCID: https://orcid.org/0000-0001-8308-3419 \\ Universidade do Estado do Pará, Brasil \\ E-mail: drphmf@yahoo.com.br \\ Sarah Lais Rocha \\ ORCID: https://orcid.org/0000-0002-3753-9507 \\ Universidade do Estado do Pará, Brasil \\ Faculdade Carajás, Brasil \\ E-mail: sarahlaisrocha@gmail.com \\ Lucas Henrique de Amorim Lima \\ ORCID: https://orcid.org/0000-0001-6757-6360 \\ Universidade do Estado do Pará, Brasil \\ E-mail: lucasheenrique2000@gmail.com
}

\begin{abstract}
Resumo
Objetivo: investigar, na literatura nacional e internacional, as características das condutas de pacientes com Transtorno Depressivo Maior no âmbito da Atenção Primária à Saúde. Métodos: trata-se de uma Revisão Integrativa de Literatura (RIL), com uma busca sensível e específica nas bases de dados: Biblioteca Virtual em Saúde (BVS), PubMed e Cochrane Library, através dos termos e seus correlatos: "Terapêutica", "Transtorno Depressivo Maior", "Atenção Primária à Saúde" e "Médicos da Família". Resultados: Foram identificados 518 estudos, após aplicação dos critérios de elegibilidade 20 estudos foram incluídos na síntese qualitativa, que foram publicados em oito países diferentes de 1995 até 2020, apresentando população total de 35.590 pacientes com transtorno depressivo maior. Discussão: percebeu-se que os estudos abordavam em intervenção medidas farmacológicas, não farmacológicas, como psicoterapia e educação em saúde, assim como medidas alternativas, como gestão de cuidados. Conclusão: os métodos utilizados apresentaram eficácia no tratamento da depressão, porém poucos estudos sobre a temática foram abordados no Brasil.
\end{abstract}

Palavras-chave: Atenção primária à saúde; Transtorno depressivo maior; Terapêutica.

\begin{abstract}
Objective: to investigate, in the national and international literature, the characteristics of the behavior of patients with Major Depressive Disorder in the scope of Primary Health Care. Methods: this is an Integrative Literature Review (IRL), with a sensitive and specific search in the databases. data: Virtual Health Library (VHL), PubMed and Cochrane Library, through the terms and their correlates: "Therapeutics", "Major Depressive Disorder", "Primary Health Care" and "Family Doctors". Results: We identified 518 studies, after applying the eligibility criteria 20 studies included in the qualitative synthesis, which were published in eight different countries from 1995 to 2020, changed total population of 35,590 patients with major depressive disorder. Discussion: evasion studies addressed pharmacological and nonpharmacological measures in intervention, such as psychotherapy and health education, as well as alternative measures, such as care management. Conclusion: the methods used were dissipation in the treatment of depression, but few studies on the subject were used in Brazil.
\end{abstract}

Keywords: Primary health care; Depressive disorder, Major; Therapeutics.

\section{Resumen}

Objetivo: investigar, en la literatura nacional e internacional, las características del comportamiento de los pacientes con Trastorno Depresivo Mayor en el ámbito de la Atención Primaria de Salud. Métodos: se trata de una Revisión de Literatura Integrativa (IRL), con una búsqueda sensible y específica en las bases de datos. datos: Biblioteca Virtual en Salud (BVS), PubMed y Biblioteca Cochrane, a través de los términos y sus correlatos: "Terapéutica", "Trastorno 
depresivo mayor", “Atención primaria de salud” y "Médicos de familia”. Resultados: Se identificaron 518 estudios, luego de aplicar los criterios de elegibilidad 20 estudios incluidos en la síntesis cualitativa, que fueron publicados en ocho países diferentes entre 1995 y 2020, cambiaron la población total de 35.590 pacientes con trastorno depresivo mayor. Discusión: los estudios de evasión abordaron medidas farmacológicas y no farmacológicas en la intervención, como la psicoterapia y la educación para la salud, así como medidas alternativas, como la gestión del cuidado. Conclusión: los métodos utilizados fueron la disipación en el tratamiento de la depresión, pero en Brasil se utilizaron pocos estudios sobre el tema.

Palabras clave: Atención primaria de salud; Trastorno depresivo mayor; Terapéutica.

\section{Introdução}

O Transtorno Depressivo Maior é uma psicopatia recorrente que afeta 265 milhões de pessoas no mundo (Organização Mundial da Saúde, 2021). No Brasil, de acordo com a Pesquisa Nacional em Saúde (PNS), aproximadamente 16,3 milhões de pessoas apresentam essa condição psicológica, apresentando-se como um importante transtorno mental no cenário nacional, diante do crescimento de casos diagnosticados nos últimos anos. Ainda, a depressão afeta principalmente mulheres e pessoas com mais de 60 anos, com maior prevalência nas regiões Sul e Sudeste (IBGE, 2020).

As causas da depressão maior são variadas e enigmáticas, existe uma complexidade entre fatores biológicos, psicológicos e sociais na sua prevalência, eventos adversos também podem desencadeá-la. É um transtorno mental responsável pelo aumento da carga global de outras doenças e de incapacidades, através de suas manifestações clínicas, que se caracterizam por tristeza persistente, perturbações do sono, apetite e concentração, bem como diminuição do interesse em práticas agradáveis, seus efeitos podem se perpetuar por anos e afetar o funcionamento social, configurando-se como fator de risco para o suicídio (Organização Pan-Americana de Saúde, 2021; Guimarães et al., 2019).

Nessa perspectiva, o diagnóstico e tratamento adequado se torna fundamental para melhoria da qualidade de vida do paciente com sofrimento psíquico. No Brasil, cerca 29,7\% dos casos são diagnosticados em Unidades Básicas de Saúde (UBS), no contexto do serviço público, a Atenção Básica é o local em que mais se diagnostica e trata a depressão, uma vez que se apresenta como porta de entrada para o Sistema Único de Saúde (SUS) (IBGE, 2020). Desse modo, a Estratégia de Saúde da Família (ESF) e a atuação do médico da família são importantes para o manejo adequado dessa psicopatia (Daré, Caponi, 2016).

Nesse sentido, existem diversas terapêuticas farmacológicas e psicológicas capazes de amenizar os sintomas do transtorno depressivo maior. Todavia, diversos fatores podem interferir na adesão e eficácia do tratamento, além da disponibilidade diminuída em regiões que não são cobertas pelos serviços de saúde (OPAS, 2021). No estudo de Motta et al. (2017) diversas abordagens são usadas no contexto da Atenção Primária à Saúde para o manejo da depressão, embora em sua maioria sejam sob a perspectiva biológica, relacionada às práticas tradicionais, com as formas de atenção que contemplem os recursos psicossociais serem pouco utilizadas, evidenciando que a integralidade dos serviços necessita ser mais consolidadas na atenção primária.

Dessa forma, é imprescindível a compreensão das condutas utilizadas na atenção primária e sua eficácia para tratar a depressão, de modo a evidenciar as diversas práticas capazes de minimizar de forma segura seus sintomas, para que possam ser implementadas garantindo maior integridade dos serviços. Portanto, o objetivo deste estudo foi investigar, na literatura nacional e internacional, as características das condutas de pacientes com Transtorno Depressivo Maior no âmbito da Atenção Primária à Saúde.

\section{Metodologia}

Na perspectiva de entendimento sobre o manejo de pacientes com transtorno depressivo maior no cenário da atenção primária em saúde, foi realizada uma Revisão Integrativa de Literatura (RIL), que proporciona a síntese de conhecimento e aplicação dos resultados de estudos significativos na prática (Souza, Silva, Carvalho, 2010). Desse modo, seguindo as fases no 
estudo de Souza, Silva \& Carvalho (2010), foi percorrido o seguinte percurso metodológico: elaboração da pesquisa norteadora, busca ou amostragem na literatura, coleta de dados, análise crítica dos estudos incluídos, discussão dos resultados e, por fim, apresentação da revisão integrativa.

\subsection{Elaboração da pergunta norteadora}

Para determinar os objetivos do estudo foi considerada a seguinte pergunta norteadora: "Quais as principais condutas dos pacientes com Depressão Maior atendidas na Atenção Primária à Saúde?”. Dessa forma, foi possível determinar o perfil dos pacientes atendidos e analisar as terapêuticas farmacológicas, psicossociais e alternativas utilizadas no contexto da Atenção Básica. Ainda, para facilitar a busca foi definida a estratégia PICO conforme Figura 1. A Estratégia PICO de pesquisa é uma ferramenta utilizada na prática baseada em evidências, de modo a subsidiar a decisão clínica, uma vez que facilita a construção da pergunta de pesquisa e da revisão de literatura (Santos, Pimenta, Nobre, 2007).

Figura 1. Descrição da estratégia PICO.

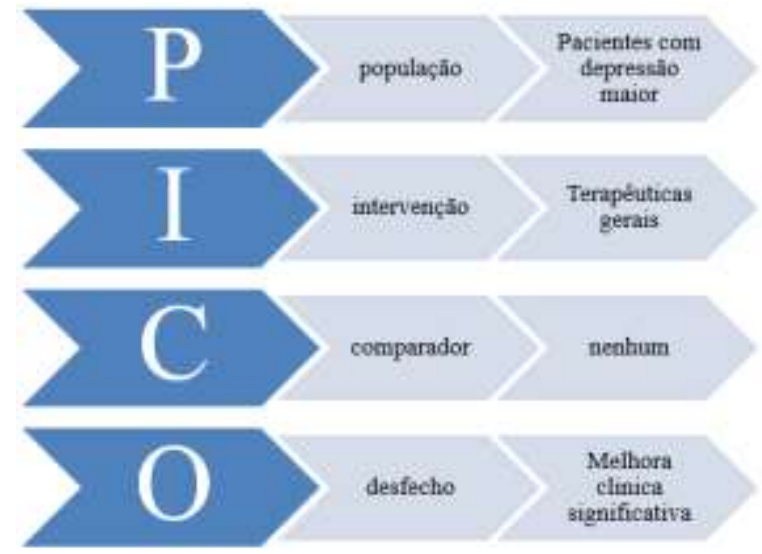

Fonte: Autores (2021).

\subsection{Busca ou Amostragem na Literatura}

Foi realizada uma busca sensível e específica nas bases de dados: Biblioteca Virtual em Saúde (BVS), sendo usado os termos e seus correlatos, de acordo com o Descritores em Ciências da Saúde (DeCS). Cochrane Library e PubMed com os termos e os correlatos baseados no Medical Subject Headings (MeSH).

\subsubsection{Estratégia de Busca Biblioteca Virtual em Saúde}

((Terapêutica) OR (Ação Terapêutica) OR (Ações Terapêuticas) OR (Medida Terapêutica) OR (Medidas Terapêuticas) OR (Procedimento Curativo) OR (Procedimento de Terapia) OR (Procedimento de Tratamento) OR (Procedimento Terapêutico) OR (Procedimentos Curativos) OR (Procedimentos de Terapia) OR (Procedimentos de Tratamento) OR (Procedimentos Terapêuticos) OR (Propriedade Terapêtica) OR (Terapia) OR (Terapias) OR (Tratamento) OR (Tratamentos)) AND ((Transtorno Depressivo Maior) OR (Depressão Involutiva) OR (Melancolia Involutiva) OR (Parafrenia Involutiva) OR (Psicose Involutiva)) AND ((Atenção Primária à Saúde) OR (Atendimento Básico) OR (Atendimento Primário) OR (Atendimento Primário de Saúde) OR (Atenção Básica) OR (Atenção Básica à Saúde) OR (Atenção Básica de Saúde) OR (Atenção Primária) OR (Atenção Primária de Saúde) OR (Atenção Primária em Saúde) OR (Cuidado de Saúde Primário) OR (Cuidado Primário de Saúde) OR (Cuidados de Saúde Primários) OR (Cuidados Primários) OR (Cuidados Primários à Saúde) OR (Cuidados Primários de Saúde) OR (Primeiro Nível de Assistência) OR (Primeiro Nível de Atendimento) OR (Primeiro Nível de Atenção) OR (Primeiro Nível de Atenção à Saúde) OR (Primeiro Nível de Cuidado) OR (Primeiro Nível de Cuidados)) AND (Médicos de 
Família)

\subsubsection{Estratégia de Busca Cochrane Library}

((Therapeutics) OR (Therapeutic) OR (Therapy) OR (Therapies) OR (Treatment) OR (Treatments)) AND ((Depressive Disorders, Major) OR (Major Depressive Disorders) OR (Major Depressive Disorder) OR (Paraphrenia, Involutional) OR (Involutional Paraphrenia) OR (Involutional Paraphrenias) OR (Paraphrenias, Involutional) OR (Psychosis, Involutional) OR (Involutional Psychoses) OR (Involutional Psychosis) OR (Psychoses, Involutional) OR (Depression, Involutional) OR (Involutional Depression) OR (Melancholia, Involutional) OR (Involutional Melancholia)) AND ((Primary Health Care) OR (Care, Primary Health) OR (Health Care, Primary) OR (Primary Healthcare) OR (Healthcare, Primary) OR (Primary Care) OR (Care, Primary)) AND ((Physicians, Family) OR (Family Physician) OR (Family Physicians) OR (Physician, Family))

\subsubsection{Estratégia de Busca PubMed}

(((c(((Therapeutic) OR (Therapy)) OR (Therapies)) OR (Treatment)) OR (Treatments)) AND Disorders, Major) OR (Major Depressive Disorders)) OR (Major Depressive Disorder)) OR (Paraphrenia, Involutional)) OR (Involutional Paraphrenia)) OR (Involutional Paraphrenias)) OR (Paraphrenias, Involutional)) OR (Psychosis, Involutional)) OR (Involutional Psychoses)) OR (Involutional Psychosis)) OR (Psychoses, Involutional)) OR (Depression, Involutional)) OR (Involutional Depression)) OR (Melancholia, Involutional)) OR (Involutional Melancholia))) AND (((((((Primary Health Care) OR (Care, Primary Health)) OR (Health Care, Primary)) OR (Primary Healthcare)) OR (Healthcare, Primary)) OR (Primary Care)) OR (Care, Primary))) AND ((((Physicians, Family) OR (Family Physician)) OR (Family Physicians)) OR (Physician, Family))

\subsection{Coleta dos Dados}

Após a busca nas bases de dados, todos os artigos foram exportados para o software Rayyan QCRI (http://rayyan.qcri.org), após a identificação das duplicatas, dois pesquisadores independentes realizaram a triagem inicial com a deliberação por um terceiro pesquisador em casos de conflitos. Essa ferramenta é bastante utilizada em revisões de literatura, uma vez que agiliza a triagem de resumos e títulos por meio de um processo de semi automação de modo rápido e eficiente (Ouzzani et al. 2016).

Após essa triagem inicial, os estudos com potenciais para RIL foram lidos na íntegra e tiveram os dados extraídos para uma planilha do Microsoft Excel, com informações sobre os estudos (título, nome do periódico, nome dos autores, ano de publicação, país de publicação, principais resultados, intervenção e desfechos) e sobre a população (número, sexo e idade).

\subsection{Análise Crítica dos Estudos Incluídos}

Esses estudos com potenciais foram analisados na íntegra de forma rigorosa, respeitando os seguintes critérios de elegibilidade: estudos observacionais completos na íntegra publicados em periódicos e que apresentaram população adulta, sendo descartados todos os artigos que não respondiam aos objetivos, que não se adequaram ao desenho de estudo, pesquisas de natureza não humana e artigos duplicados.

\subsection{Discussão dos Resultados}

Após a leitura integral foi realizada uma síntese com os principais dados evidenciados dos estudos incluídos, comparando os resultados e agrupando os estudos que abordassem a mesma terapêutica, de modo a identificar possíveis lacunas do conhecimento. 


\section{Resultados}

\subsection{Seleção dos estudos}

Após a busca foram identificados 518 estudos, sendo 449 da PubMed, 59 da Cochrane Library e 11 da Biblioteca Virtual em Saúde. Desses, 38 estudos foram removidos como duplicatas, restando 480 estudos, após a triagem de títulos e resumos 94 artigos foram selecionados para leitura integral, nessa etapa apenas 20 estudos foram considerados com potencial para a RIL. A figura 2 apresenta o fluxograma PRISMA, descrevendo todas as etapas da seleção.

Figura 2. Fluxograma PRISMA.
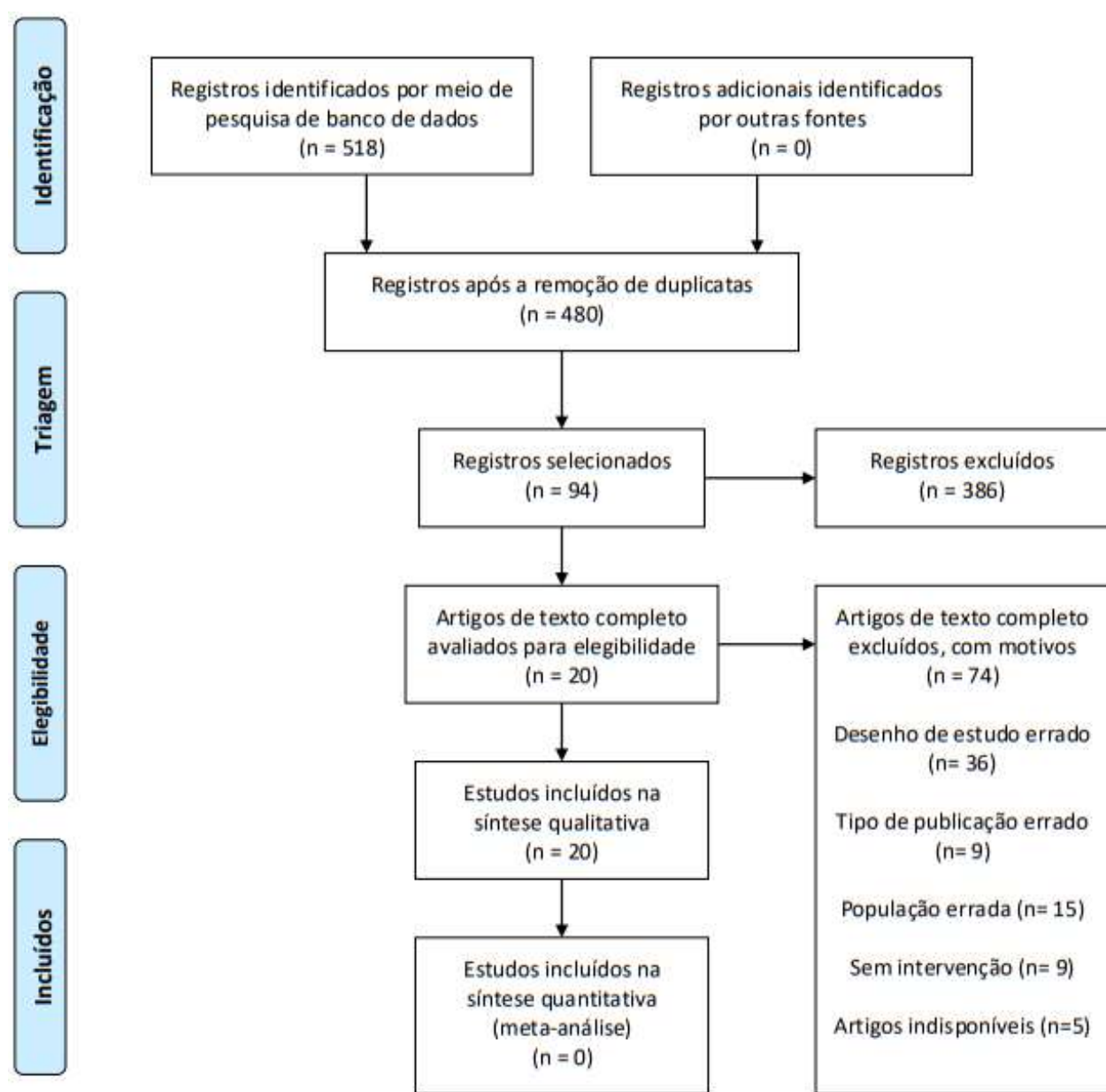

Fonte: Autores (2021).

\subsection{Caracterização dos Estudos Incluídos}

Os estudos incluídos na síntese qualitativa foram publicados entre os anos1995 e 2020, em oito países diferentes, sendo: (11) Estados Unidos da América, (02) Alemanha, (02) Canadá, (01) Bósnia e Herzegovina, (01) Croácia, (01) Holanda, (01) Inglaterra e (01) Noruega. Todos no idioma Inglês, apresentando uma população total de 35.590 pacientes diagnosticados com Transtorno Depressivo Maior que receberam algum tipo de terapêutica, conforme apresentado no Quadro 1. 
Quadro 1. Características dos Estudos.

\begin{tabular}{|c|c|c|c|c|}
\hline Título & Autor/ano & País & População & Intervenção \\
\hline $\begin{array}{l}\text { A collaborative model for thetreatmentofdepression in } \\
\text { homeboundelders }\end{array}$ & Knight et al., 2008 & EUA & 179 & Gestão Colaborativa \\
\hline $\begin{array}{l}\text { A methodthatdramatically improves } \\
\text { patientadherencetodepressiontreatment }\end{array}$ & Ruoff, 2005 & EUA & 103 & Educação em Saúde \\
\hline Antidepressant treatment outcomes in family medicine & Prasko et al., 2019 & $\begin{array}{c}\text { Bósnia e } \\
\text { Herzegovina }\end{array}$ & 508 & Farmacológico \\
\hline $\begin{array}{l}\text { Case management for depression by health care } \\
\text { assistants in small primary care practices: a cluster } \\
\text { randomized trial }\end{array}$ & Gensichen et al., 2009 & Alemanha & 626 & Gestão Colaborativa \\
\hline $\begin{array}{c}\text { Clinical relevance of vilazodone treatment in patients } \\
\text { with major depressive disorder: categorical } \\
\text { improvement in symptoms }\end{array}$ & Culpepper et al., 2014 & EUA & 864 & Farmacológico \\
\hline $\begin{array}{l}\text { Collaborative care management of late-life depression } \\
\text { in the primary care setting: a randomized controlled } \\
\text { trial }\end{array}$ & Unützer et al., 2002 & EUA & 1801 & Gestão Colaborativa \\
\hline $\begin{array}{l}\text { Collaborative management to achieve treatment } \\
\text { guidelines. Impact on depression in primary care }\end{array}$ & Katon et al., 1995 & EUA & 217 & Gestão Colaborativa \\
\hline $\begin{array}{c}\text { Combinatorial pharmacogenomics and improved } \\
\text { patient outcomes in depression: Treatment by primary } \\
\text { care physicians or psychiatrists }\end{array}$ & Tanner et al., 2018 & Canadá & 1871 & $\begin{array}{l}\text { Farmacogenômica } \\
\text { Combinatória }\end{array}$ \\
\hline $\begin{array}{l}\text { Comparison of depression treatment among different } \\
\text { age groups in primary care setting }\end{array}$ & $\begin{array}{l}\text { Blažeković-Milaković } \\
\text { et al., } 2011\end{array}$ & Croácia & 17290 & Farmacológico \\
\hline $\begin{array}{l}\text { Improvement in functional outcomes with adjunctive } \\
\text { Aripiprazole versus placebo in major depressive } \\
\text { disorder: a pooled post Hoc analysis of } 3 \text { short-term } \\
\text { studies }\end{array}$ & Fabian et al., 2012 & EUA & 1088 & Farmacológico \\
\hline $\begin{array}{c}\text { Intensive psychotherapy and case management for } \\
\text { Karen refugees with major depression in primary care: } \\
\text { a pragmatic randomized control trial }\end{array}$ & $\begin{array}{l}\text { Northwood et al., } \\
\qquad 2020\end{array}$ & Inglaterra & 214 & Gestão Colaborativa \\
\hline $\begin{array}{l}\text { Long-term effects of a collaborative care intervention } \\
\text { on process of care in family practices in Germany: a } \\
\text { 24-month follow-up study of a cluster randomized } \\
\text { controlled trial }\end{array}$ & Petersen et al., 2014 & Alemanha & 626 & Gestão Colaborativa \\
\hline $\begin{array}{l}\text { Physical Exercise for Late-Life Depression: } \\
\text { Customizing an Intervention for Primary Care }\end{array}$ & Zanetidou et al., 2017 & EUA & 121 & ExercícioFísico \\
\hline $\begin{array}{l}\text { Primary care physician use of cognitive behavioral } \\
\text { techniques with depressed patients }\end{array}$ & Robinson et al., 1995 & EUA & 155 & Psicoterapia \\
\hline $\begin{array}{l}\text { Stepped collaborative depression care: primary care } \\
\text { results before and after implementation of a stepped } \\
\text { collaborative depression programme }\end{array}$ & Gidding et al., 2014 & Holanda & 7979 & Gestão Colaborativa \\
\hline $\begin{array}{l}\text { St John's wort or sertraline? Randomized controlled } \\
\text { trial in primary care }\end{array}$ & Van-Gurp et al., 2002 & Canadá & 87 & Fitoterápico \\
\hline $\begin{array}{l}\text { The education of depressed primary care patients: what } \\
\text { do patients think of interactive booklets and a video? }\end{array}$ & Robinson et al., 1997 & EUA & 108 & Educação em Saúde \\
\hline $\begin{array}{l}\text { The effect of a primary care practice-based depression } \\
\text { intervention on mortality in older adults: a randomized } \\
\text { trial }\end{array}$ & Gallo et al., 2007 & EUA & 1226 & Gestão Colaborativa \\
\hline $\begin{array}{l}\text { The Norwegian naturalistic treatment study of } \\
\text { depression in general practice (NORDEP)-I: } \\
\text { randomized double-blind study }\end{array}$ & Malt et al., 1999 & Noruega & 372 & Psicoterapia \\
\hline $\begin{array}{l}\text { The role of the primary care physician in patients' } \\
\text { adherence to antidepressant therapy }\end{array}$ & Lin et al., 1995 & EUA & 155 & Educação em Saúde \\
\hline
\end{tabular}

Fonte: Autores (2021). 


\section{Discussão}

Após a leitura integral dos estudos incluídos foi possível identificar as principais medidas adotadas por médicos da família, no contexto da atenção primária à saúde, sendo agrupado em lacunas do conhecimento de modo a agrupar possíveis evidências. Nesse contexto, percebeu-se que os estudos abordavam em intervenção medidas farmacológicas, não farmacológicas e medidas alternativas.

\subsection{Medidas farmacológicas}

Os estudos de Prasko et al. (2019) Culpepper et al. (2014) Blažeković-Milaković et al. (2011) e Fabian et al. (2012) avaliaram terapias farmacológicas e suas eficácias no tratamento da depressão maior. Fabian et al. (2012) avaliou o efeito do aripiprazol adjuvante à terapia antidepressiva e concluiu que seu uso, comparado ao placebo produziu melhora significativa nos sintomas, bem como maiores mudanças de níveis graves/moderados para leves. Esse medicamento é um antipsicótico que atua como agonista em diversos receptores no sistema nervoso (Fabian et al., 2012).

Por sua vez, Culpepper et al. (2014) comparou o uso de vilazodona $40 \mathrm{mg}$ ou placebo, evidenciando que pacientes com depressão maior moderados ou graves fazendo uso da vilazodonaevoluíram para sintomas leves ou remissão dos sintomas. Na maioria dos sintomas, como tristeza profunda, fadiga e distúrbios do sono, os pacientes tratados com vilazodona apresentaram $50 \%$ mais probabilidade de mudar para os sintomas mínimos ou nenhum sintoma, sugerindo que pacientes tratados com vilazodona apresenta melhorias clinicamente relevante dos sintomas.

Prasko et al. (2019) comparou a prevalência da depressão de acordo com o medicamento de escolha, seus resultados apontam que a maioria dos pacientes são tratados com Inibidores Seletivos da Recaptação de Serotonina (ISRS), porém a probabilidade de recorrência dos sintomas é alta (90\%). Desse modo, os antidepressivos tricíclicos apresentam resultados melhores, porém seus efeitos adversos são preocupantes. Ainda, o estudo de Blažeković-Milaković et al. (2011) também comparou o uso dos antidepressivos, mostrando que os ISRS foram fármacos bastantes utilizados, assim como os Benzodiazepínicos, no geral a combinação de farmacoterapia e apoio/psicoterapia foi o tratamento com melhores resultados. Dessa forma, o tratamento da depressão requer que o médico da família tenha boas habilidades e conhecimentos sobre o uso da farmacoterapia apropriada.

Tanner et al. (2018) relata que o uso da farmacogenômica combinatória para orientar a seleção de medicamentos está associado a melhores respostas, com resultados significativamente melhores entre os pacientes tratados por médicos da família. Enquanto, Van-Gurp et al. (2002) comparou a mudança na gravidade dos sintomas depressivos e as reações adversas em pacientes tratados na atenção básica com sertralina e com erva de São João, os resultados mostraram que a erva de são joão apresenta menos efeitos colaterais o tornando uma boa primeira escolha para essa população de paciente. Esse estudo evidencia que o tratamento da depressão pode ter outras alternativas, diferentes das que estamos familiarizados.

\subsection{Medidas não farmacológicas}

Nessa perspectiva, Ruoff (2005), Robinson et al. (1997) e Lin et al. (1995) utilizaram como terapêutica medidas de educação em saúde, de modo a orientar explicar para o paciente questões relacionadas à psicopatia e ao tratamento, com objetivo de aumentar a adesão ao tratamento e consequentemente melhora clínica. Por exemplo, o estudo de Ruof (2005) apresentou um fluxograma de manejo da depressão baseada em instruções médicas, educação do paciente e acompanhamento diligente, enquanto Robinson et al. (1997) comparou os resultados dos pacientes que receberam tratamento e um pacote de material educacional com pacientes que não receberam o material, evidenciando que a orientação do paciente melhorou significativamente o quadro clínico, por fim, Lin et al. (1997) demonstrou que médicos da atenção primária podem melhorar a adesão ao tratamento por meio de mensagens educacionais simples e especificas integradas durante as consultas, de modo a 
orientar o paciente sobre os efeitos da terapia antidepressiva, sintomas e reações adversas. Em todos os estudos que usaram medidas de educativas, os pacientes tiveram melhora clinica dos sintomas da depressão maior.

Robinson et al. (1995) e Malt et al. (1999) avaliaram os efeitos da psicoterapia, evidenciando que a combinação do aconselhamento, apoio emocional e tratamento psicológico simples junto com a terapia medicamentos foi mais eficaz do que apenas uma terapêutica isolada. Desse modo, as orientações do médico da família sobre planejamento de atividades prazerosas, resolução de problemas, planejamento de atividades que aumentem a confiança e diminua os pensamentos depressivos foram fundamentais para alcançar os melhores resultados. Por fim, Zanetidou et al. (2017) avaliou a pratica clínica de intervenções baseadas no exercício físico, e mostrou que a combinação dessa prática com o tratamento farmacológico melhora os resultados clínicos dos pacientes com depressão maior, visto que essa atividade pode ser uma atividade prazerosa.

\subsection{Medidas alternativas}

Os estudos de Knight et al. (2008), Gensichen et al. (2009), Unützer et al. (2002) Katon et al. (1995), Northwood et al. (2020) Petersen et al. (2014) Gidding et al. (2014) e Gallo et al. (2007) avaliaram a gestão de cuidados como medida terapêutica no transtorno depressivo maior.

Essa abordagem caracteriza-se pela colaboração entre os médicos de saúde da família, pacientes e médicos especialistas, de modo a desenvolver uma aliança terapêutica e um plano de tratamento personalizado incluindo as necessidades e preferências do paciente, bem como a atendimento escalonado com outros profissionais da unidade de saúde, de modo a garantir o acompanhamento proativo e monitorização dos resultados (Unützer et al.2002; Gidding et al.2014).

A atenção primária à saúde compreende como o primeiro nível de atenção a diagnosticar e conduzir o tratamento da depressão, e essa intervenção multifacetada, garante maior interdisciplinaridade e minimização das limitações desse manejo, assim como a satisfação e adesão ao tratamento (Katon et al. 1995), provocando uma diminuição no número de encaminhamentos para um nível de maior complexidade e a melhora clínica do paciente (Gidding et al.2014).

Northwood et al. (2020) comparou o desfecho de pacientes que foram submetidos à gestão de cuidados e psicoterapia com o atendimento usual para depressão, e concluiu que houve uma redução considerável dos sintomas e melhoria do funcionamento social nos pacientes submetidos à essa intervenção multiprofissional. O mesmo resultado encontrado no estudo de Gensichen et al. (2009) e Unützer et al. (2002), que evidenciou que pacientes submetidos à gestão de cuidados tiveram uma redução dos sintomas maior que 50\% no primeiro ano, em comparação com $19 \%$ dos pacientes que receberam apenas o tratamento habitual.

Por sua vez, Katon et al. (1995) relatou que $74 \%$ dos pacientes apresentaram melhora de 50\% maior comparada ao grupo dos cuidados habituais, assim como maior adesão ao tratamento farmacológico e diminuição da gravidade ao longo do tempo. Knight et al. (2008) evidenciou uma melhoria significativa em pacientes idosos tratados com o modelo colaborativo, visto que essa psicopatia é bastante prevalente nessa faixa etária. Para Gallo et al. (2007) os pacientes com depressão em práticas de gerenciamento colaborativo tinham menos chances de morrer do que aqueles em práticas de tratamentos usuais.

Desse modo, o modelo de gestão colaborativa se mostra viável no contexto da atenção básica e significativamente mais eficaz do que o tratamento usual para a depressão, garantindo melhora significativa dos sintomas, maior adesão ao tratamento, diminuição do nível de internação, complicações e morte (Knight et al., 2008; Gensichen et al., 2009; Unützer et al., 2002; Katon et al., 1995; Northwood et al., 2020; Petersen et al., 2014; Gidding et al., 2014; Gallo et al., 2007)..

\section{Conclusão}

Nesse contexto, é evidente que diversas intervenções para tratar o transtorno depressivo maior são utilizadas no contexto da atenção primária à saúde. O médico da família tem papel crucial no desenvolvimento dessas medidas, o tratamento 
farmacológico com métodos alternativos de cuidados, bem como orientação ao paciente, com equipe multidisciplinar se mostra eficaz na melhora significativa dos sintomas depressivos. Embora, seja necessário que estudos futuros pesquisem sobre a combinação das terapêuticas identificadas nessa revisão.

Os estudos apresentam qualidade metodológica e intervenções eficazes, entretanto, foram encontrados poucos estudos sobre essa temática nos últimos cinco anos, bem como na América Latina, fazendo-se necessário incentivar a produção de estudos futuros sobre a temática, sobretudo no Brasil.

\section{Referências}

Culpepper, L., Mathews, M., Ghori, R., \& Edwards, J. (2014). Clinical relevance of vilazodone treatment in patients with major depressive disorder: categorical improvement in symptoms. 16(1). https://www.cochranelibrary.com/central/doi/10.1002/central/CN-00984342/full

Lin, E. H, et al., (1995). The role of the primary care physician in patients' adherence to antidepressant therapy. Medical Care, 33(1), 67-74. https://pubmed.ncbi.nlm.nih.gov/7823648/

Fabian, T. J., Cain, Z. J., Ammerman, D., Eudicone, J. M., Tang, Y., Rollin, L. M., Forbes, R. A., Berman, R. M., \& Baker, R. A. (2012). Improvement in functional outcomes with adjunctive Aripiprazole versus placebo in major depressive disorder: a pooled post Hoc analysis of 3 short-term studies. 14(6). https://www.cochranelibrary.com/central/doi/10.1002/central/CN-00908729/full

Ruoff, G. (2005). A method that dramatically improves patient adherence to depression treatment. The Journal of Family Practice, 54(10), 846-852. https://pubmed.ncbi.nlm.nih.gov/16202372/

Gallo, J. J., Bogner, H. R., Morales, K. H., Post, E. P., Lin, J. Y., \& Bruce, M. L. (2007). The effect of a primary care practice-based depression intervention on mortality in older adults: a randomized trial. 146(10), 689-698. https://www.cochranelibrary.com/central/doi/10.1002/central/CN-00588850/full

Gidding, L. G., Spigt, M. G., \& Dinant, G.-J. (2014). Stepped collaborative depression care: primary care results before and after implementation of a stepped collaborative depression programme. Fam Pract, 31(2), 180-192. https://dx.doi.org/10.1093/fampra/cmt072

Gensichen, J. et al., (2009). Case management for depression by health care assistants in small primary care practices: a cluster randomized trial. Annals of Internal Medicine, 151(6), 369-378. https://pubmed.ncbi.nlm.nih.gov/19755362/

Unützer, J. et al., (2002). Collaborative care management of late-life depression in the primary care setting: a randomized controlled trial. JAMA, 288(22), 28362845. https://pubmed.ncbi.nlm.nih.gov/12472325/

Tanner, J. A., et al., (2018). Combinatorial pharmacogenomics and improved patient outcomes in depression: Treatment by primary care physicians or psychiatrists. Journal of Psychiatric Research, 104, 157-162. https://pubmed.ncbi.nlm.nih.gov/30081389/

Petersen, J. J., et al., (2014). Long-term effects of a collaborative care intervention on process of care in family practices in Germany: a 24 -month follow-up study of a cluster randomized controlled trial. General Hospital Psychiatry, 36(6), 570-574. https://pubmed.ncbi.nlm.nih.gov/25135191/

Katon, W., Von Korff, M., Lin, E., Walker, E., Simon, G. E., Bush, T., Robinson, P., \& Russo, J. (1995). Collaborative management to achieve treatment guidelines. Impact on depression in primary care. 273(13), 1026-1031. https://www.cochranelibrary.com/central/doi/10.1002/central/CN-00112197/full

Knight, M. M,, \& EA, H. (2008). A collaborative model for the treatment of depression in homebound elders. Issues in Mental Health Nursing, 29(9), 974-991. https://pubmed.ncbi.nlm.nih.gov/18770102/

Northwood, A. K., Vukovich, M. M., Beckman, A., Walter, J. P., Josiah, N., Hudak, L., O’Donnell Burrows, K., Letts, J. P., \& Danner, C. C. (2020). Intensive psychotherapy and case management for Karen refugees with major depression in primary care: a pragmatic randomized control trial. $21(1)$, 17. https://www.cochranelibrary.com/central/doi/10.1002/central/CN-02087764/full

Robinson, P. et al., (1995). Primary care physician use of cognitive behavioral techniques with depressed patients. The Journal of Family Practice, 40(4), 352357. https://pubmed.ncbi.nlm.nih.gov/7699348/

Robinson, P., Katon, W., Von Korff, M., Bush, T., Simon, G., Lin, E., \& Walker, E. (1997). The education of depressed primary care patients: what do patients think of interactive booklets and a video? 44(6), 562-571. https://www.cochranelibrary.com/central/doi/10.1002/central/CN-00140717/full

Blažeković-Milaković, S. et al., (2011). Comparison of depression treatment among different age groups in primary care setting. Psychiatria Danubina, 23(2), 183-188. https://pubmed.ncbi.nlm.nih.gov/21685858/

Prasko, S. et al., (2019). Antidepressant treatment outcomes in family medicine. Medicinski Glasnik : Official Publication of the Medical Association of ZenicaDoboj Canton, Bosnia and Herzegovina, 16(2). https://pubmed.ncbi.nlm.nih.gov/31257837/

Zanetidou. S. et al., (2017). Physical Exercise for Late-Life Depression: Customizing an Intervention for Primary Care. Journal of the American Geriatrics Society, 65(2), 348-355. https://pubmed.ncbi.nlm.nih.gov/27869986/

Malt, U. F. et al., (1999). The Norwegian naturalistic treatment study of depression in general practice (NORDEP)-I: randomised double blind study. BMJ (Clinical Research Ed.), 318(7192), 1180-1184. https://pubmed.ncbi.nlm.nih.gov/10221945/

Van Gurp, G., Meterissian, G. B., Haiek, L. N., McCusker, J., \& Bellavance, F. (2002). St John's wort or sertraline? Randomized controlled trial in primary care. 48, 905-912. https://www.cochranelibrary.com/central/doi/10.1002/central/CN-00389488/full 
Research, Society and Development, v. 11, n. 2, e7211225539, 2022

(CC BY 4.0) | ISSN 2525-3409 | DOI: http://dx.doi.org/10.33448/rsd-v11i2.25539

Instituto Brasileiro de Geografia e Estatística (2020). Pesquisa Nacional de Saúde. Acervo IBGE. https://biblioteca.ibge.gov.br/visualizacao/livros/liv101764.pdf

Guimarães, L. A. et al. (2019). Sintomas depressivos e fatores associados em idosos residentes em instituição de longa permanência. Ciência \& Saúde Coletiva [online]. 24(9) 3275-3282. <https://doi.org/10.1590/1413-81232018249.30942017>.

Motta, C. C. L; Moré, C. L. O. O.; Nunes, C. H. S. S. (2017). O atendimento psicológico ao paciente com diagnóstico de depressão na Atenção Básica. Ciência \& Saúde Coletiva, 22(3):911-920.

OPAS (2021). Depressão, Tópicos. Organização Pan-americana de Saúde. https://www.paho.org/pt/topicos/depressao.

Organização Mundial da Saúde (2021). Depression. https://www.who.int/health-topics/depression.

Ouzzani et al (2016). Rayyan — a web and mobile app for systematic reviews. Systematic Reviews 5:210, DOI: 10.1186/s13643-016-0384-4. https://systematicreviewsjournal.biomedcentral.com/articles/10.1186/s13643-016-0384-4

Santos, Cristina Mamédio da Costa, Pimenta, Cibele Andrucioli de Mattos e Nobre, Moacyr Roberto Cuce (2007). The PICO strategy for the research question construction and evidence search. Revista Latino-Americana de Enfermagem [online]. 15(3):508-511 <https://doi.org/10.1590/S0104-11692007000300023>.

Souza, M. T. de, Silva, M. D. da, \& Carvalho, R. de. (2010). Integrative review: what is it? How to do it? Einstein (São Paulo), 8(1), $102-106$. 